\title{
Atopic Dermatitis Topical Therapies: Study of YouTube Videos as a Source of Patient Information
}

\author{
Amylee Martin, BS; Akshitha Thatiparthi, BS; Jeffrey Liu, BS; Jashin J. Wu, MD
}

\section{PRACTICE POINTS}

- YouTube is a readily accessible resource for educating patients about topical treatments for atopic dermatitis.

- Although professional source videos comprised a larger percentage of the videos included within our study, patient experience videos had a higher number of views and engagement.

- Twenty-one percent (19/92) of the videos examined in our study discussed topical steroid withdrawal, and the majority of them were patient experience videos.

To the Editor:

Atopic dermatitis (eczema) affects approximately $20 \%$ of children worldwide. ${ }^{1}$ In atopic dermatitis management, patient education is crucial for optimal outcomes. ${ }^{2}$ The COVID-19 pandemic has impacted patient-physician interactions. To ensure safety of patients and physicians, visits may have been canceled, postponed, or conducted virtually, leaving less time for discussion and questions. ${ }^{3}$ As a consequence, patients may seek information about atopic dermatitis from alternative sources, including YouTube videos. We performed a cross-sectional study to analyze YouTube videos about topical treatments for atopic dermatitis.

During the week of July 16, 2020, we performed 4 private browser YouTube searches with default filters using the following terms: eczema topicals, eczema topical treatments, atopic dermatitis topicals, and atopic dermatitis topical treatments. For video selection, we defined topical treatments as topical corticosteroids, topical calcineurin inhibitors, crisaborole, emollients, wet wraps, and any prospective treatment topically administered. For each of the 4 searches, 2 researchers (A.M. and A.T.) independently examined the top 75 videos, yielding a total of 300 videos. Of them, 98 videos were duplicates, 19 videos were not about atopic dermatitis, and 91 videos were not about topical treatments, leaving a total of 92 videos for analysis (Figure 1).

For the 92 included videos, the length; upload year; number of views, likes, dislikes, and comments; interaction ratio (IR)(the sum of likes, dislikes, and comments divided by the number of views); and video content were determined. The videos were placed into mutually exclusive categories as follows: (1) patient experience, defined as a video about

\footnotetext{
Ms. Martin is from the School of Medicine, University of California, Riverside. Ms. Thatiparthi is from the College of Osteopathic Medicine of the Pacific, Western University of Health Sciences, Pomona, California. Mr. Liu is from the Keck School of Medicine, University of Southern California, Los Angeles. Dr. Wu is from Dermatology Research and Education Foundation, Irvine, California.

Ms. Martin, Ms. Thatiparthi, and Mr. Liu report no conflict of interest. Dr. Wu is or has been an investigator, consultant, or speaker for AbbVie, Almirall, Amgen, Arcutis Biotherapeutics, Aristea Therapeutics Inc., Boehringer Ingelheim, Bristol-Myers Squibb, Dermavant Sciences, Inc, Dr. Reddy's Laboratories, Eli Lilly and Company, Galderma, Janssen Pharmaceuticals, LEO Pharma, Mindera, Novartis, Regeneron Pharmaceuticals, Sanofi Genzyme, SOLIUS, Sun Pharmaceutical Industries Ltd, UCB, Valeant Pharmaceuticals North America LLC, and Zerigo Health. The eTable is available in the Appendix online at www.mdedge.com/dermatology. 


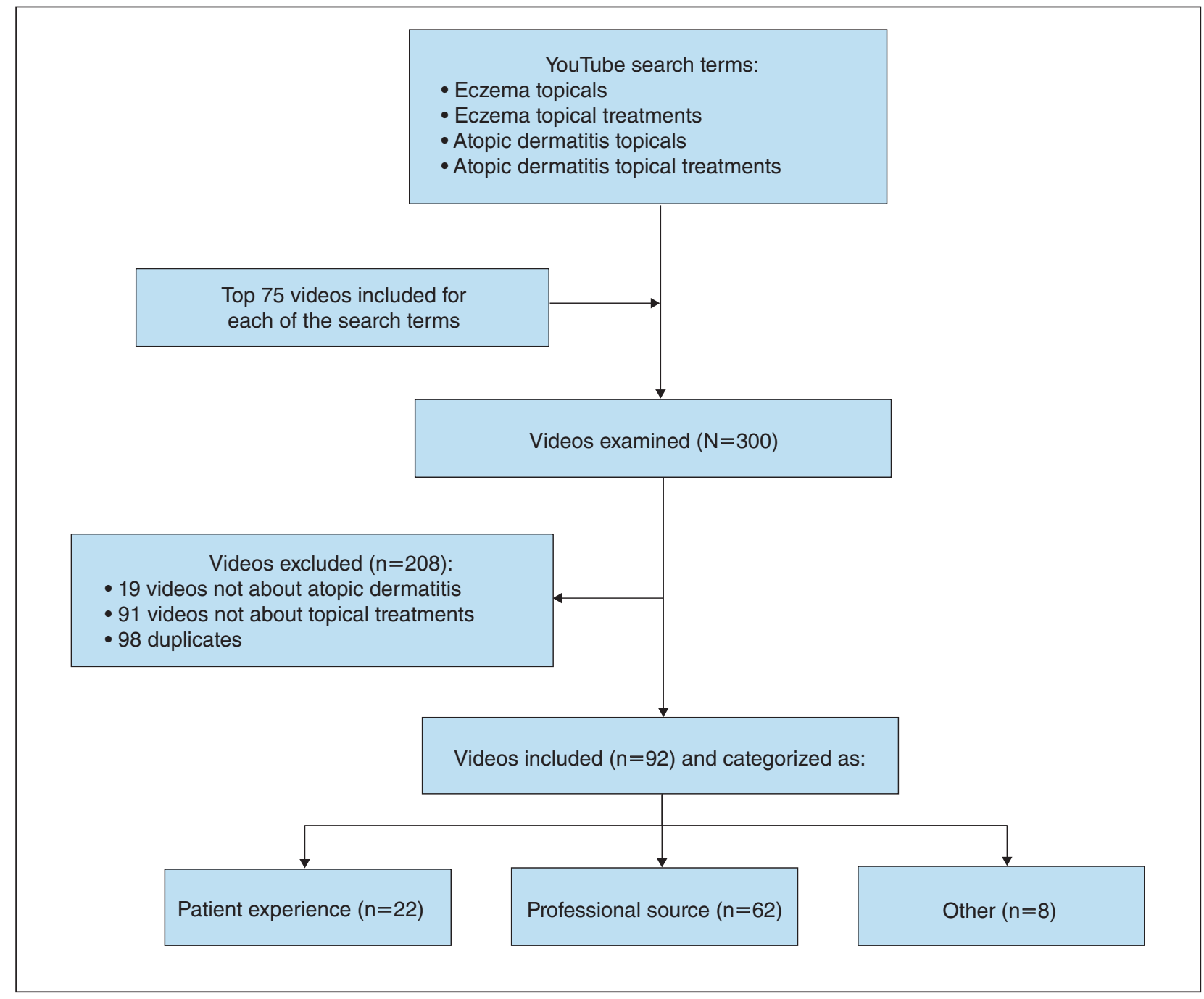

FIGURE 1. Visual representation of the YouTube video selection process.

patient perspective; (2) professional source, defined as a video featuring a physician, physician extender, pharmacist, or scientist, or produced by a formal organization; or (3) other. The DISCERN Instrument was used for grading the reliability and quality of the 92 included videos. This instrument consists of 16 questions with the responses rated on a scale of 1 to $5 .{ }^{4}$ For analysis of DISCERN scores, patient experience and other videos were grouped together as nonprofessional source videos. A 2-sample $t$-test was used to compare DISCERN scores between professional source and nonprofessional source videos.

Most videos were uploaded in $2017(n=19), 2018$ $(n=23)$, and $2019(n=25)$, but 20 were uploaded in 20122016 and 5 were uploaded in 2020. The 92 videos had a mean length of 8 minutes and 35 seconds (range, 30 seconds to 62 minutes and 23 seconds).

Patient experience videos accounted for $23.9 \%$ $(n=22)$ of videos. These videos discussed topical steroid withdrawal $(\mathrm{TSW})(\mathrm{n}=16)$, instructions for making emollients $(n=2)$, and treatment successes $(n=4)$. Professional source videos represented $67.4 \%(n=62)$ of videos. Of them, $40.3 \%(n=25)$ were physician oriented, defined as having extensive medical terminology or qualifying for continuing medical education credit. Three $(4.8 \%)$ of the professional source videos were sponsored by a drug company. Other constituted the remaining $8.7 \%(n=8)$ of videos.

Patient experience videos had more views (median views [interquartile range], 6865 [10,307]) and higher engagement (median IR [interquartile range], 0.038 [0.022]) than professional source videos (views: median views [interquartile range], 1052.5 [10,610.5]; engagement: median IR [interquartile range], 0.006 [0.008]).

Although less popular, professional source videos had a significantly higher DISCERN overall quality rating score (question 16) compared to those categorized as nonprofessional source (3.92 vs $1.53 ; P<.001)$. In contrast, 


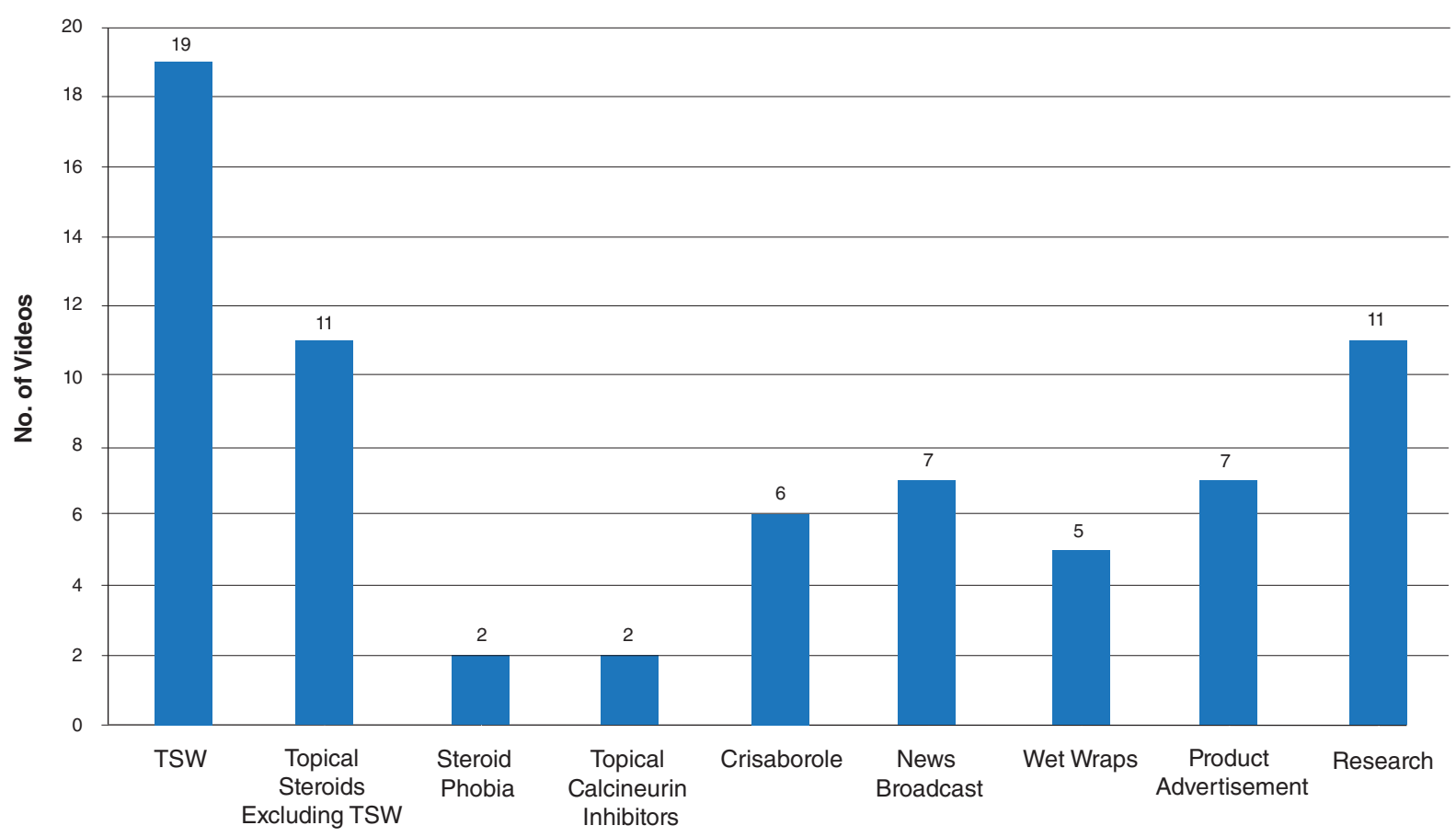

Atopic Dermatitis Topical Treatments

FIGURE 2. Video categories for atopic dermatitis topical treatments. Featured categories are not mutually exclusive or comprehensivee. TSW indicates topical steroid withdrawal.

nonprofessional source videos scored significantly higher on the quality-of-life question (question 13) compared to professional source videos (3.90 vs $2.56 ; P<.001$ ) (eTable). (Three professional source videos were removed from YouTube before DISCERN scores could be assigned.)

Notably, 20.7\% ( $n=19)$ of the 92 videos discussed TSW, and most of them were patient experiences $(n=16)$. Other categories included topical steroids excluding TSW $(n=11)$, steroid phobia $(n=2)$, topical calcineurin inhibitors $(n=2)$, crisaborole $(n=6)$, news broadcast $(n=7)$, wet wraps $(n=5)$, product advertisement $(n=7)$, and research $(n=11)$ (Figure 2). Interestingly, there were no videos focusing on the calcineurin inhibitor black box warning.

Similar to prior studies, our results indicate preference for patient-generated videos over videos produced by or including a professional source. ${ }^{5}$ Additionally, only 3 of 19 videos about TSW were from a professional source, increasing the potential for patient misconceptions about topical corticosteroids. Future studies should examine the educational impact of patient-generated videos as well as features that make the patient experience videos more desirable for viewing.

\section{REFERENCES}

1. Mueller SM, Hongler VNS, Jungo P, et al. Fiction, falsehoods, and few facts: cross-sectional study on the content-related quality of atopic eczema-related videos on YouTube. J Med Internet Res. 2020;22:e15599. doi:10.2196/15599

2. Torres T, Ferreira EO, Gonçalo M, et al. Update on atopic dermatitis. Acta Med Port. 2019;32:606-613. doi:10.20344/amp.11963

3. Vogler SA, Lightner AL. Rethinking how we care for our patients in a time of social distancing during the COVID-19 pandemic. Br J Surg. 2020;107:937-939. doi:10.1002/bjs.11636

4. The DISCERN Instrument. discern online. Accessed January 22, 2021. http://www.discern.org.uk/discern_instrument.php

5. Pithadia DJ, Reynolds KA, Lee EB, et al. Dupilumab for atopic dermatitis: what are patients learning on YouTube? [published online April 16, 2020]. J Dermatolog Treat. doi:10.1080/09546634.2020.1755418 


\section{APPENDIX}

\section{DISCERN Scores for YouTube Videos Discussing Atopic Dermatitis Topical Treatments}

\begin{tabular}{|c|c|c|c|}
\hline \multirow[b]{2}{*}{ DISCERN question } & \multicolumn{2}{|c|}{ DISCERN score, mean (SD) } & \multirow[b]{2}{*}{$P$ value $^{\mathrm{c}}$} \\
\hline & $\begin{array}{l}\text { Nonprofessional } \\
\text { source videos }(n=30)^{a}\end{array}$ & $\begin{array}{l}\text { Professional source } \\
\text { videos }(n=62)^{b}\end{array}$ & \\
\hline \multicolumn{4}{|l|}{ Reliability $^{d}$} \\
\hline 1. Are the aims clear? & $4.33(1.12)$ & $3.86(1.09)$ & .061 \\
\hline 2. Does it achieve its aims? & $4.86(0.44)$ & $4.67(0.47)$ & .075 \\
\hline 3. Is it relevant? & $2.20(0.66)$ & $4.81(0.57)$ & $<.001$ \\
\hline $\begin{array}{l}\text { 4. Is it clear what sources of information were used to compile } \\
\text { the publication? }\end{array}$ & $1.17(0.46)$ & $2.63(1.77)$ & $<.001$ \\
\hline $\begin{array}{l}\text { 5. Is it clear when the information used or reported in the } \\
\text { publication was produced? }\end{array}$ & $1.03(0.18)$ & $2.31(1.66)$ & $<.001$ \\
\hline 6. Is it balanced and unbiased? & $1.40(0.62)$ & $4.36(1.17)$ & $<.001$ \\
\hline $\begin{array}{l}\text { 7. Does it provide details of additional sources of support } \\
\text { and information? }\end{array}$ & $2.30(1.26)$ & $2.12(1.15)$ & .497 \\
\hline 8. Does it refer to areas of uncertainty? & $2.00(1.08)$ & $3.15(1.73)$ & .001 \\
\hline \multicolumn{4}{|l|}{ Quality of Information } \\
\hline 9. Does it describe how each treatment works? & $2.53(1.57)$ & $4.02(1.33)$ & $<.001$ \\
\hline 10. Does it describe the benefits of each treatment? & $3.60(1.77)$ & $4.17(1.57)$ & .125 \\
\hline 11. Does it describe the risks of each treatment? & $2.97(1.90)$ & $3.15(1.86)$ & .660 \\
\hline $\begin{array}{l}\text { 12. Does it describe what would happen if no treatment } \\
\text { is used? }\end{array}$ & $2.30(1.49)$ & $1.83(1.29)$ & .127 \\
\hline $\begin{array}{l}\text { 13. Does it describe how the treatment choices affect overall } \\
\text { quality of life? }\end{array}$ & $3.90(1.45)$ & $2.56(1.58)$ & $<.001$ \\
\hline $\begin{array}{l}\text { 14. Is it clear that there may be more than } 1 \text { possible } \\
\text { treatment choice? }\end{array}$ & $2.60(1.57)$ & $3.36(1.65)$ & .041 \\
\hline 15. Does it provide support for shared decision-making? & $1.43(0.97)$ & $3.07(1.64)$ & $<.001$ \\
\hline Overall Rating of Publication & & & \\
\hline 16. Overall quality rating ${ }^{9}$ & $1.53(0.63)$ & $3.92(0.93)$ & $<.001$ \\
\hline
\end{tabular}

ancludes patient experience videos $(n=22)$ and other videos $(n=8)$.

${ }^{\mathrm{b}}$ Three professional source videos were removed from YouTube before DISCERN scores could be assigned.

${ }^{c} P$ value calculated using 2 -sample $t$-test.

$\mathrm{d} 1=$ no, $3=$ partially, $5=$ yes.

e $1=$ no, $3=$ partially, $5=$ yes.

${ }^{f} 1=$ serious or extensive shortcomings, $3=$ potentially important but no serious shortcomings, $5=$ minimal shortcomings.

${ }^{9}$ Question from the DISCERN instrument: "Based on the answers to all of the above questions, rate the overall quality of the publication as a source of information about treatment questions." 